\title{
Can reporting of adverse drug reactions create safer systems while improving health data?
}

\author{
Corinne Hohl MD MHSc, Joel R. Lexchin MSc MD, Ellen Balka PhD \\ CMAJ Podcasts: author interview at soundcloud.com/cmajpodcasts/drug-reaction
}

See also www.cmaj.ca/lookup/doi/10.1503/cmaj.109-5028

$\mathrm{O}$ n Nov. 6, 2014, the Canadian government passed Bill C-17 (Vanessa's Law), aimed at strengthening the powers of government to recall a drug that is shown to have harmful effects. ${ }^{1}$ The bill is expected to strengthen postmarket surveillance and research, and allow the Minister of Health to compel the release of proprietary information and drug safety studies when a marketed health product is suspected of posing a risk to health. However, its success in achieving its desired impact will in part depend on how the regulations influence documentation of adverse drug reactions.

The bill currently does not require health care providers to document serious adverse drug reactions, but mandates that health care institutions report all documented serious reactions. Therefore, lack of documentation of adverse drug reactions by health care providers may minimize any gains that might be achieved from compulsory reporting, unless better documentation rates can be achieved.

In a prospective study published in 2008, $5 \%$ of emergency department visits to one Canadian tertiary care centre were due to adverse drug reactions. $^{2}$ Of these visits, $37 \%$ resulted in hospital admission, thus meeting Health Canada's definition of serious adverse drug reactions. ${ }^{2}$ Data from the United States have confirmed that adverse drug reactions are among the most common admitting diagnoses. ${ }^{3}$ If representative, these data suggest that each acute care hospital in Canada should be documenting and reporting thousands of adverse drug reactions each year to fulfill the goal of Bill C-17.

Although large numbers of patients receive treatment in acute care hospitals for adverse drug reactions, few events are reported to Canada Vigilance (Health Canada's online and paper-based reporting platform that health care professionals and consumers are expected to access) even after they are recognized. Wiktorowicz and colleagues estimate that less than $5 \%$ of reactions are reported, even in jurisdictions where reporting is mandatory. ${ }^{4}$ Extremely serious reactions (e.g., toxic epidermal necrolysis) are underreported in Canada by as much as $96 \% .^{5}$ Although administrative datasets are invaluable in providing population-level data to answer some questions about drug safety and effectiveness, only a fraction of adverse drug reactions are identifiable within them unless broad coding algorithms are used that have low specificity. ${ }^{6}$ Similarly, medical-record review using trigger methods has low sensitivity for the identification of adverse drug reactions.?

According to qualitative research, many systems developed or used to support documentation of adverse drug reactions (e.g., computer-based patient-safety learning systems or paper-based forms) are poorly fitted to clinical practice, timeconsuming to complete and not integrated into clinical processes. Consequently, clinicians do not use them. ${ }^{8}$ In a recent study, as soon as clinicians had to exit a computerized patient chart to complete a report, their willingness to document adverse drug reactions declined. ${ }^{8}$ Clinicians never chose to document reactions within paper or electronic systems that requested voluminous (and often duplicate) data unless those data could support immediate and short-term patient safety goals by allowing the retrieval of meaningful patientlevel data to inform care. ${ }^{8}$ In a systematic review, none of 105 existing reporting systems examined

\section{KEY POINTS}

- Bill C-17 (Vanessa's Law) was passed on Nov. 6, 2014, in an attempt to bolster drug safety by strengthening postmarket surveillance and research in Canada.

- The bill currently does not require health care providers to document serious adverse drug reactions, but mandates that health care institutions report all documented serious reactions; unless documentation of adverse events improves, the potential of the law to do good will be small.

- Existing reporting platforms are poorly fitted to clinical practice and are not well used by clinicians; few events are documented and reported to Canada Vigilance, despite large numbers of patients receiving treatment in acute care hospitals for adverse drug reactions.

- A new reporting framework that revolves around patients and their interaction with providers must be developed if Bill C-17 is to succeed in improving available health data on adverse drug reactions. 
could use reported information to generate patientlevel alerts to assist clinicians in ensuring that absolutely contraindicated culprit drugs were not represcribed or redispensed, a well-documented cause of readmissions. ${ }^{9}$

Existing systems further deterred clinicians from documenting adverse drug reactions because the systems failed to reflect the clinical processes through which adverse drug reactions were identified, and because of the ambiguity and uncertainty about what level of suspected reaction should be reported. ${ }^{8}$ Identifying a patient as experiencing an adverse drug reaction typically spans time and locations (e.g., emergency department and inpatient wards) and is not a discrete event involving a single clinician. Therefore, reporting infrastructures must be accessible to multiple clinicians across multiple settings. If clinicians were asked (e.g., by software) whether an adverse drug reaction occurred before the clinician made a definitive diagnosis, clinicians did not complete a report. Care providers also highlighted the need for information contained in reports of adverse drug reactions to be modifiable (e.g., if the initial cause of delirium was suspected to be a drug, but this was subsequently confirmed as false). ${ }^{8}$ Unless these issues are addressed, enacting the law with existing systems is doomed to fail even as reporting to Health Canada is mandated.

A new framework for documenting and reporting adverse drug reactions must be developed if Vanessa's Law is to succeed, because improved documentation is a prerequisite to more comprehensive reporting. Reports for adverse drug reactions must be simple and quick to complete, and support clinical decisions at the point of care. Although studies of successfully implemented reporting systems are sparse, Yen and colleagues ${ }^{10}$ reported increases in the number of events documented and decreases in the number of preventable events following introduction of an electronic reporting system. Key factors for successful implementation are the system itself, extensive involvement by clinicians (i.e., physicians, pharmacists and nurses), and the implementation process. Platforms must be intuitive, user-friendly and easily accessible within electronic medical records, and must motivate documentation by making patient care safer. Patient- and medication-level data can then be integrated into administrative data, anonymized for surveillance and research purposes, and reported to external agencies, thus enabling Health Canada to receive timely and representative data as a by-product of safer care. This could transform postmarket surveillance in Canada.

Work organization, staffing mixes, data abstraction processes and the computing environment may influence optimal designs for data collection and implementation. The likelihood of successful implementation will be improved by recognition that variability exists within and between health care institutions. The same reporting processes and systems do not need to be implemented in all locations. Rather, data must be meaningfully aggregated across systems. Regulations to support Vanessa's Law should specify a common data set and definitions, but allow institutions some local control in how data are captured and reported.

Vanessa's Law can strengthen Canada's drug safety environment. However, it needs to be strengthened through specific regulations that mandate the implementation of reporting infrastructure that is seamlessly integrated into electronic medical record systems. With provincial and territorial governments responsible for health care delivery, strong leadership will be required provincially and within health care organizations to draft regulations in collaboration with Health Canada to ensure that documentation processes for adverse drug reactions are patient-focused and provider-centred, and generate higher quality representative data for improved safety.

\section{References}

1. Herder M, Gibson E, Graham J, et al. Regulating prescription drugs for patient safety: Does Bill C-17 go far enough? CMAJ 2014; 186:E287

2. Zed PJ, Abu-Laban RB, Balen RM, et al. Incidence, severity and preventability of medication-related visits to the emergency department: a prospective study. CMAJ 2008;178:1563-9.

3. Budnitz DS, Lovegrove MC, Shehab N, et al. Emergency hospitalizations for adverse drug events in older americans. $N$ Engl $J$ Med 2011;365:2002-12.

4. Wiktorowicz M, Lexchin J, Paterson M, et al. Research networks involved in post-market pharmacosurveillance in the United States, United Kingdom, France, New Zealand, Australia, Norway and European Union: lessons for Canada. Edmonton: Canadian Patient Safety Insitute; 2008.

5. Mittmann N, Knowles S, Gomez M, et al. Evaluation of the extent of under-reporting of serious adverse drug reactions: the case of toxic epidermal necrolysis. Drug Saf 2004;27:477-87.

6. Hohl CM, Kuramoto L, Yu E, et al. Evaluating adverse drug event reporting in administrative data from emergency departments: a validation study. BMC Health Serv Res 2013;13:473-84.

7. Karpov A, Mok C, Parcero C, et al. Validation of trigger tools and the National Electronic Injury Surveillance System-Cooperative Adverse Drug Event Surveillance Project (NEISS-CADES) Algorithms for identification of adverse drug events within emergency department health records. CJEM 2014;15:S21.

8. Peddie D, Hohl C, Bailey C, et al. Challenges care providers face documenting adverse drug events: an observational study. Vancouver: Quality Forum; 2015.

9. Bailey C, Peddie D, Wickham M, et al. Adverse drug event reporting: getting the message across. A systematic review of adverse drug event reporting systems. Vancouver: Quality Forum; 2015

10. Yen Y, Kuo L, Hsu M, et al. Evaluation of the electronic adverse drug event management system. J Experiment Clin Med. 2010;26:287-91.

Affiliations: Emergency Department (Hohl), Vancouver General Hospital, Vancouver, BC; School of Health Policy and Management (Lexchin), York University, Toronto, Ont.; School of Communications (Balka), Simon Fraser University, Vancouver, BC

Contributors: All of the authors wrote the article, gave final approval of the version to be published and agreed to act as guarantors of the work. 